\title{
Superior mesenteric artery syndrome and acute pancreatitis in a boy with eating disorder: a case report
}

\author{
Toru Watanabe ${ }^{1}$, Motoi Fujita ${ }^{2}$, Yutaka Hirayama ${ }^{3}$, Yasushi Iinuma ${ }^{3}$ \\ ${ }^{1}$ Departments of Pediatrics, Niigata City General Hospital, Niigata, Japan; \\ ${ }^{2}$ Department of Pediatrics, Niigata National Hospital, National Hospital Organization, Kashiwazaki, Japan; \\ ${ }^{3}$ Pediatric Surgery, Niigata City General Hospital, Niigata, Japan. \\ Email: twata@hosp.niigata.niigata.jp
}

Received 2 October 2011; revised 12 November 2011; accepted 20 November 2011.

\begin{abstract}
Eating disorders are an important cause of physical and psychosocial disturbances. Gastrointestinal complications in eating disorders are common and include pancreatitis and superior mesenteric artery syndrome (SMAS). However, only two patients who simultaneously suffered from both SMAS and pancreatitis in the setting of an eating disorder have ever been reported in the literature. We describe here a patient with eating disorder not otherwise specified (ED-NOS) who exhibited both SMAS and pancreatitis concomitantly. An 11-year-old boy with ED-NOS had presented with the sudden onset of recurrent bilious vomiting, abdominal distension and abdominal pain. Laboratory and imaging studies revealed that the patient had SMAS and acute pancreatitis. Gastric decompression and intravenous infusion of fluids promptly improved his abdominal symptoms and laboratory data. This is the first case report of a patient with ED-NOS who simultaneously suffered from SMAS and pancreatitis in the pediatric literature. Since SMAS and pancreatitis can both cause patients to be severely ill, these diseases should be considered in patients with eating disorders who exhibit abdominal symptoms such as vomiting or abdominal pain.
\end{abstract}

Keywords: Eating Disorder Not Otherwise Specified; Superior Mesenteric Artery Syndrome; Pancreatitis; Gastrointestinal Complications

\section{INTRODUCTION}

Eating disorders are an important cause of physical and psychosocial disturbances, which primarily affect adolescent females and can have life-threatening medical consequences [1]. They are categorized into three disorders including anorexia nervosa, bulimia nervosa and eating disorder not otherwise specified (ED-NOS) in the Diagnostic and Statistical Manual of Mental Disorders, fourth edition (DSM-IV) [2]. ED-NOS includes patients with clearly aberrant eating patterns and weight management habits who do not meet the criteria for anorexia nervosa or bulimia nervosa [2].

The physical abnormalities in eating disorders are secondary to the patients' disturbed eating habits and their compromised nutritional state, which may induce endocrine, cardiovascular, hematological and gastrointestinal system dysfunctions as well as other metabolic abnormalities [2].

Gastrointestinal complications in eating disorders are common and include parotid enlargement with hyperamylasemia, dysphagia, gastric dilatation due to delayed gastric emptying, constipation, pancreatic abnormalities [1] and superior mesenteric artery syndrome (SMAS) [3]. However, only two patients who simultaneously suffered from both SMAS and pancreatitis have ever been reported in eating disorders $[4,5]$. We describe here a patient with ED-NOS who exhibited both SMAS and pancreatitis concomitantly, the first case report of a patient with these diseases in the pediatric literature.

\section{PATIENT REPORT}

An 11-year-old boy was admitted to our hospital with a one-day history of recurrent bilious vomiting, abdominal distension and abdominal pain. The patient had been diagnosed with ED-NOS 1 month before because of 3-month history of excessive exercising and restricted intake without severe body image disturbance that led to a $6 \mathrm{~kg}$ weight loss; in addition, the patient denied the serious nature of his illness. He had a past medical history notable for Kawasaki syndrome complicated by coronary artery aneurysms at 3 years of age, which was treated with high-dose gamma globulin therapy and a duodenal ulcer at 8 years of age. His older sister has also suffered from anorexia nervosa and selective mutism 
since 10 years of age.

Physical examination on admission revealed extreme emaciation with a weight of $27 \mathrm{~kg}$ (37\% below normal for age/height), height of $150 \mathrm{~cm}$, body mass index (BMI) of $12 \mathrm{~kg} / \mathrm{m}^{2}$, bradycardia (pulse rate was $40 / \mathrm{bpm}$ ), abdominal distention with hypoactive bowel sounds and diffuse abdominal tenderness to palpation without guarding or rebound.

Laboratory studies disclosed metabolic alkalosis with respiratory compensation ( $\mathrm{pH} 7.455, \mathrm{HCO}_{3} 36.3 \mathrm{mmHg}$, and PCO2 $52.8 \mathrm{mmHg}$ ), hypernatremia (Na $150 \mathrm{mEq} / \mathrm{l}$ ), elevated blood urea nitrogen $(51.4 \mathrm{mg} / \mathrm{dl})$ and serum creatinine $(1.10 \mathrm{mg} / \mathrm{dl})$ levels, and hyperamylasenemia (484 IU/l) with a predominant increase of pancreatic isozymes. Serum lipase (608 U/l [normal 13 - $49 \mathrm{U} / \mathrm{l}]$ ), trypsin $(900 \mathrm{ng} / \mathrm{ml}$ [normal $110-460 \mathrm{ng} / \mathrm{ml}])$ and elastase-1 (1400 ng/ml [normal 100 - $400 \mathrm{ng} / \mathrm{dl}$ ]) levels were also increased. Liver function tests, white blood cell counts, blood glucose levels and concentrations of Creactive protein were within the normal ranges.

Because an abdominal X-ray demonstrated an extremely dilated stomach, the patient underwent nasogastric tube decompression with drainage of $500 \mathrm{ml}$ of bilious fluid, which improved his abdominal signs and symptoms promptly. A post-contrast abdominal computed tomography after nasogastric tube insertion revealed loss of intra-abdominal adipose tissue, reduction of the aortomesenteric distance (4 mm [normal 10 - $28 \mathrm{~mm}$ ) and the aorto-mesenteric angle (13 [normal 38 - 65]) [6] without any abnormal findings of the other organs including pancreas (Figure 1). An upper gastrointestinal tract contrast-medium study demonstrated an abrupt vertical cut off of the third portion of the duodenum (Figure 2).

The patient was diagnosed as having ED-NOS complicated by SMAS and pancreatitis, and he underwent

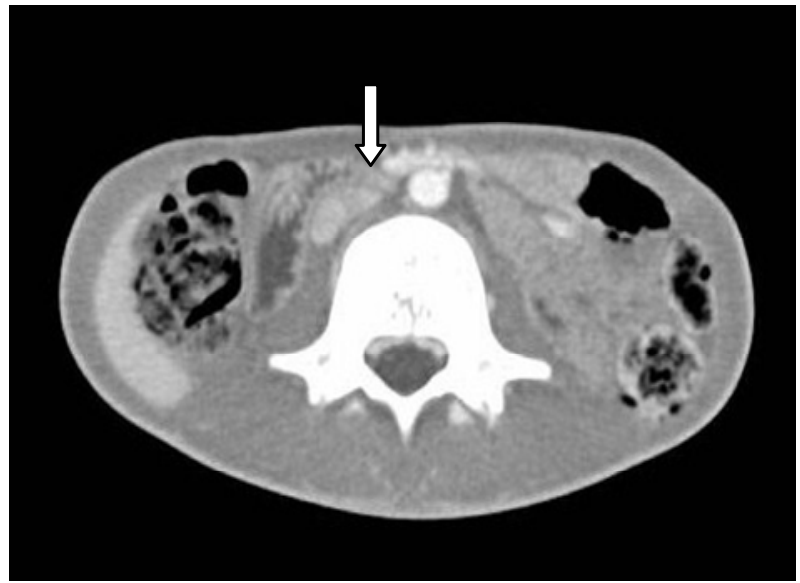

Figure 1. Axial post-contrast computed tomography of the abdomen demonstrating duodenal obstruction between the superior mesenteric artery and the aorta (arrow).

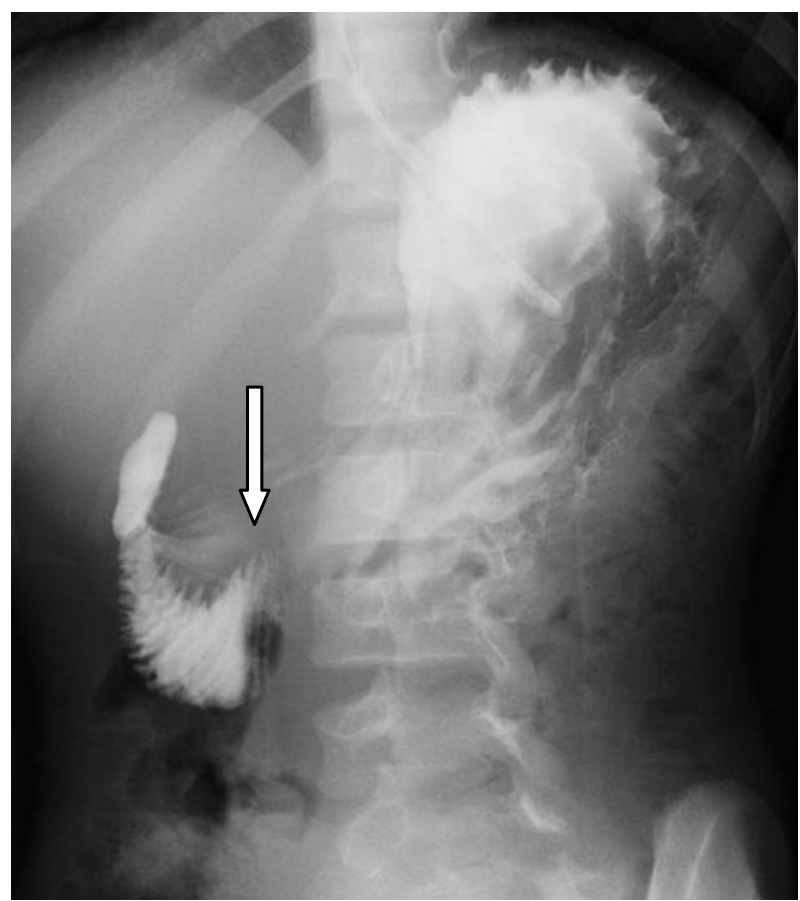

Figure 2. Upper gastrointestinal tract contrast-medium study showing an abrupt cut off the third portion of duodenum (arrow).

intravenous fluid infusions and enteral feedings by nasojejunal tube. His serum levels of amylase and lipase decreased into the normal range one week following the initiation of treatment, although his weight and nutritional status did not improved. The other abnormal laboratory tests also normalized within two weeks.

Because the patient had exhibited psychiatric symptoms including irritability and profound anxiety, he was transferred to the eating disorders unit. He underwent psychiatric care in addition to the nutritional therapy, which improved his condition gradually. The patient became well with body weight of $37 \mathrm{Kg}$ (14\% below normal for age/height) two months after being transferred to the unit and was discharged.

\section{DISCUSSION}

SMAS and pancreatitis are well-known gastrointestinal complications of eating disorders [1]. However, only two patients who concurrently suffered from both SMAS and pancreatitis similarly to our patient have been reported in eating disorders.

Keane et al. presented a 16-year-old girl with anorexia nervosa who developed acute gastric dilation and duodenal ileus due to SMAS and acute pancreatitis following rapid refeeding [4]. Gwee et al. recently described a 17-year-old girl with anorexia nervosa who exhibited acute abdominal symptoms following rapid refeeding, and was diagnosed with SMAS and acute pancreatitis [5]. 
Unlike these two cases, our patient with ED-NOS developed SMAS and pancreatitis simultaneously without rapid refeeding.

SMAS involves the entrapment and obstruction of the third part of the duodenum between the SMA and aorta due to the narrowing of the angle between both vessels [6]. The cause of this narrowing is related to loss of intra-abdominal adipose tissue, which may result from eating disorders [6]. Symptoms of SMAS can be acute or chronic, with intermittent exacerbation [6]. Our patient developed acute-onset SMAS. Although SMAS has commonly been reported to present as a chronic condition in adulthood, one pediatric study reported that SMAS was more likely to present acutely, with sudden onset of symptoms related to bowel obstruction [7]. It may be difficult to distinguish whether gastrointestinal symptoms of patients with eating disorders result from the eating disorders themselves or from chronic SMAS associated with the eating disorders [3]. SMAS can precipitate and exacerbate anorexia nervosa because of nausea associated with a small bowel obstruction and conversely, anorexia nervosa prevents the patient from being willing or able to ingest adequate calories to allow the SMAS to resolve [8]. Moreover, some fatal cases of SMAS in eating disorders have been reported due to duodenal obstruction or gastric perforation [9]. Therefore patients with eating disorders should undergo imaging studies to rule out SMAS, if they exhibit abdominal symptoms such as vomiting or abdominal pain.

Pancreatitis is a potentially life-threatening illness with significant morbidity and mortality, and can occur in eating disorders [5]. Although the exact mechanism underling the development of pancreatitis in eating disorders remains unclear, two mechanisms have been suggested: pancreatic injury caused by chronic protein energy malnutrition and pancreatic regurgitation from increased duodenal pressure resulting from SMAS [4,5]. Protein energy malnutrition causes acinar cell atrophy, epithelial metaplasia and cystic dilatation of the ducts in the pancreas [10]. It is also associated with a depleted antioxidant status and subsequent susceptibility to oxidative stress and damage, and with an increase in inflammatory mediators such as TNF-alpha, IL-1 and IL-6, which have been proposed to play a role in pancreatitis [10]. Meanwhile SMAS can cause duodenal ileus and dilatation, which leads to high pancreatic duct pressure or reflux of duodenal contents into the pancreatic duct [5]. This process leads to pancreatic cell damage through autolysis thus causing the patients to develop pancreatitis [5]. Because abdominal symptoms and elevated serum amylase levels of our patient improved rapidly following only gastric decompression, an increased duodenal pressure resulting from SMAS was the most likely cause of the pancreatitis in our patient.

Our patient had a past medical history of Kawasaki syndrome complicated with coronary aneurysm and a family history of anorexia nervosa. Long-term disease condition of Kawasaki syndrome affected on the psychological condition of the patient with a family history of ED and might develop ED-NOS in this patient.

In summary, we reported a patient with ED-NOS who simultaneously suffered from SMAS and pancreatitis. Since SMAS and pancreatitis can cause patients to become severely ill, these diseases should be considered in patients with eating disorders who exhibit abdominal symptoms such as vomiting or abdominal pain.

\section{REFERENCES}

[1] McClain, C.J., Humphries, L.L., Hill, K.K. and Nickl, N.J. (1993) Gastrointestinal and nutritional aspects of eating disorders. Journal of the American College of $\mathrm{Nu}$ trition, 12, 466-474.

[2] Forman, S.F. (2010) Eating disorders: Epidemiology, pathogenesis, and clinical features. UpToDate, UpToDate Inc., Waltham.

http://www.uptodate.com/contents/eating-disorders-epide miology-pathogenesis-and-clinical-features?source=searc $\underline{\mathrm{h} \text { result\&selectedTitle }=1 \% 7 \mathrm{E} 150}$

[3] Froese, A.P., Szmuilowicz, J. and Bailey, J.D. (1978) The superior-mesenteric-artery syndrome. Cause or complication of anorexia nervosa? Canadian Psychiatric Association Journal, 23, 325-327.

[4] Keane, F.B., Fennell, J.S. and Tomkin, G.H. (1978) Acute pancreatitis, acute gastric dilatation and duodenal ileus following refeeding in anorexia nervosa. Irish Journal of Medicine Sciences, 147, 191-192. doi:10.1007/BF02939399

[5] Gwee, K. and Huang, C. (2010) Acute superior mesenteric artery syndrome and pancreatitis in anorexia nervosa. Australasian Psychiatry, 18, 523-526. doi:10.3109/10398562.2010.498885

[6] Merrett, N.D., Wilson, R.B., Cosman, P. and Biankin, A.V. (2009) Superior mesenteric artery syndrome: Diagnosis and treatment strategies. Journal of Gastrointestinal Surgery, 13, 287-292. doi:10.1007/s11605-008-0695-4

[7] Biank, V. and Werlin, S. (2006) Superior mesenteric artery syndrome in children: A 20-year experience. Journal of Pediatric Gastroenterology and Nutrition, 42, 522-525. doi:10.1097/01.mpg.0000221888.36501.f2

[8] Verhoef, P.A. and Rampal, A. (2009) Unique challenges for appropriate management of a 16-year-old girl with superior mesenteric artery syndrome as a result of anorexia nervosa: A case report. Journal of Medical Case Reports, 3, 127. doi:10.1186/1752-1947-3-127

[9] Ikegaya, H., Nakajima, M., Shintani-Ishida, K., Uemura, K. and Yoshida, K. (2006) Death due to duodenal obstruction in a patient with an eating disorder: A case report. International Journal of Eating Disorders, 39, 350- 
352. doi:10.1002/eat.20263

[10] Reddymasu, S., Banks, D.E. and Jordan, P.A. (2006)

Acute pancreatitis in a patient with malnutrition due to major depressive disorder. American Journal of Medicine, 119, 179-180. doi:10.1016/j.amjmed.2005.09.023 